\title{
Exclusive breastfeeding in Sri Lanka: problems of interpretation of reported rates
}

\author{
Suneth B Agampodi*1, Thilini C Agampodi ${ }^{1}$ and Avanthi de Silva ${ }^{2}$
} Address: ${ }^{1}$ Department of Community Medicine, Faculty of Medicine and Allied Science, Rajarata University of Sri Lanka, Saliyapura, Sri Lanka and
2Public Health Volunteer Worker, Anuradhapura, Sri Lanka

Email: Suneth B Agampodi* - sunethagampodi@yahoo.com; Thilini C Agampodi - thilinichanchala@yahoo.com; Avanthi de Silva - avanthi.desilva@gmail.com

* Corresponding author

Published: 26 November 2009

International Breastfeeding Journal 2009, 4:14 doi:10.1186/I746-4358-4-14
Received: 3 April 2009

Accepted: 26 November 2009

This article is available from: http://www.internationalbreastfeedingjournal.com/content/4/I/14

(C) 2009 Agampodi et al; licensee BioMed Central Ltd.

This is an Open Access article distributed under the terms of the Creative Commons Attribution License (http://creativecommons.org/licenses/by/2.0), which permits unrestricted use, distribution, and reproduction in any medium, provided the original work is properly cited.

\begin{abstract}
Accurate interpretation of reported breastfeeding rates is essential in understanding the true picture of a country's breastfeeding status. In Sri Lanka, where the reported exclusive breastfeeding (EBF) rate among infants aged from 0 to 5 months is $75 \%$, accurate understanding of this rate is of the utmost importance. The danger of misinterpreting the data and assuming that Sri Lanka has achieved a high EBF rate is that health workers begin to believe that no further effort should be made in this area. This is very dangerous as the potential to further improve rates of EBF will not be addressed. We discuss the interpretation of survey data and various definitions used in the relevant literature. We strongly recommend that interpretation of EBF rates should be done only after careful evaluation of the definitions and survey methods used.
\end{abstract}

\section{Introduction}

Sri Lanka declared the achievement of a 50\% improvement in rates of exclusive breastfeeding (EBF) in 2007. According to the Demographic and Health Survey (DHS) of 2007, the rate of EBF among infants aged 0-5 months in Sri Lanka was 75\% [1]. This is the highest reported rate for the South Asian region [2] and is well beyond even the ten-year target for some countries in the region. It has been highlighted by Sri Lankan Health Authorities as a major achievement in the child health program of Sri Lanka and was commended by UNICEF.

However, the figure of $75 \%$ is being misinterpreted among paediatricians, community physicians and other health workers. The DHS reports that, "among infants 0-5 months of age, the percentage who were exclusively breastfed was 75.5\%". Informal discussions with healthcare professionals in Sri Lanka indicate that the most com- mon interpretation of this figure is "75.5\% of babies in Sri Lanka are exclusively breastfed (from birth) until the completion of six months". This misinterpretation of the data is likely to be widespread in countries that use similar survey methods and reporting processes.

The purpose of this paper is to discuss the problems of definitions, measurements and interpretation of EBF rates reported in the DHS as it relates to Sri Lanka.

\section{Discussion}

\section{Definitions for estimating EBF rates}

Definitions used in breastfeeding were not agreed upon by most of the researchers for many years. Labbok discussed the need for consistency in breastfeeding definitions in 1990 [3] and called for action in 1997 [4]. In 1988 , the first international meeting related to breastfeeding definitions was held, sponsored by the Interagency 
Group for Action on Breastfeeding (IGAB). This was followed by a set of definitions published by WHO/UNICEF, which was used extensively by researchers. The WHO published an update of these definitions in 2008 [5].

For cross-sectional studies, WHO recommends estimating EBF rates based on the 24-hour recall method. In this method, information is collected on feeding practices for the day (24 hours) preceding the survey. EBF is defined by WHO as "The infant has received only breast milk from the mother or a wet nurse, or expressed breast milk, and no other liquids or solids with the exception of drops or syrups consisting of vitamins, mineral supplements, or medicines" [5].

Another widely used method of estimating EBF in surveys is the use of 'recall since birth', in which an infant is categorised as exclusively breastfed only if they have not received any food or drink other than breastmilk since birth [4]. This method requires a longer recall period, but strictly emphasises the WHO infant feeding definitions since birth.

\section{Estimating EBF rates in populations}

Defining the specific age or age categories is crucial for the measurement of EBF in populations. WHO recommends use of the indicator 'Exclusive breastfeeding under six months', defined as the 'proportion of infants 0-5 months of age who are fed exclusively with breast milk' based on the 24-hour recall method [6].

Infants $0-5$ months of age who received only breast milk during the previous day Infants $0-5$ months of age

The age range for this method is from birth to just under six months of age (0-5.99 months). It is important to note that both numerator and denominator include infants in all ages within the given range and does not represent the proportion of infants who are exclusively breastfed until just under 6 months of age. However, WHO recommends further categorization of this age range as 0-1, 2-3 and 4-5 months age groups, so that the rates are specific to the given age range.

\section{Interpretation of survey results}

Since there are various definitions of EBF and a number of ways in which the surveys can be conducted, interpreta- tion of survey results should be based on the methodology used and the age group of infants included in the study group. The Sri Lankan DHS 2007 reported, "Among infants 0-5 months of age, the percentage of exclusively breastfed infants was $75.5 \% "$. This is the average rate of breastfeeding among infants aged 0-5 months. The same survey has reported the breakdown of this rate as age specific rates. According to these rates, among infants aged 45 months, the percentage of exclusively breastfed infants was $53.4 \%$. However, in the $0-1$ month age group the percentage was 92.2 and the 2-3 months age group it was $83.7 \%$ (Table 1). The table clearly shows how EBF declines steadily from birth. Among infants aged 4-6 six months, $46.6 \%$ were not exclusively breastfed during the 24-hour period prior to the survey.

\section{Limitations of the 24-hour recall method}

The Sri Lankan DHS uses the WHO recommended 24 hour recall method to estimate EBF rates. The 24-hour recall method can always overestimate the actual EBF rate in a population. In 24-hour recall method, investigators categorize infants who were on infrequent liquids or foods, but not given those foods/liquids during the previous day as exclusively breastfed infants.

Several authors have questioned the validity of 24 hour recall method [6,7] and studies have shown that this method substantially overestimates the EBF rate, compared to the "recall since birth" method [8-10]. One of these papers reports that the difference was as high as 59\% at four months [11]. In Sri Lanka, the validity of EBF as determined using the 24-hour recall method was evaluated against the measurement of EBF since birth [12]. According to the Sri Lankan study, the proportion of infants breastfed exclusively using the 24 hour recall method was $77.4 \%$ whereas according to recall since birth it was only $49.1 \%$. Sensitivity of the WHO definition to detect non-EBF infants was 42.9\% (95\% CI: 26.5, 60.9\%) while the negative predictive value was $60.1 \%$ (95\% CI: $45.7,74.4 \%)$. This suggests that the reported rate of EBF of $53.4 \%$ for $4-5$ months infants using the 24-hour recall method might be an overestimation of the actual rate and the actual percentage of mothers practising EBF for the recommended six months period (EBF since birth to 6 months) in Sri Lanka is well below 50\%.

Table I: Breastfeeding status in Sri Lanka by age (DHS 2007) [I]

\begin{tabular}{ccc}
\hline Age in months & Number of children studied & Percentage exclusively breastfed \\
\hline $0-1$ & 172 & 92.2 \\
$2-3$ & 242 & 83.7 \\
$4-5$ & 221 & 53.4 \\
$6-8$ & 325 & 7.2 \\
\hline
\end{tabular}


A proper understanding and interpretation of survey results on breastfeeding is very important for healthcare providers to understand the true status of EBF so that they can deliver services accordingly. Accurate interpretation also affects the appropriate design of policies and programs, such as the Sri Lanka Infant Feeding Program and the Child Health Program. Although Sri Lanka has recently seen great improvements in $\mathrm{EBF}$, inaccuracies in interpretation have led to the belief that deficiencies in EBF in the population have been sufficiently addressed and that best practices have been achieved. In fact at least $46.6 \%$ of infants are not being exclusively breastfed at 4-5 months of age. Rates of EBF from birth to the completion of 6 months (0-5.99 months) is likely to be as low as $20 \%$ $[13,14]$. Further, the evaluation of definitions showed very low sensitivity of the 24 -hour recall method to detect non-EBF infants.

The danger of misinterpreting the data and assuming that Sri Lanka has achieved a high EBF rate is that health workers begin to believe that no further effort should be made in this area. This is very dangerous as the potential to further improve rates of EBF will not be addressed, thereby overlooking an important opportunity to advance child health. It should also be noted that improving EBF in Sri Lanka is not a costly and difficult process, as aptly demonstrated by a study conducted in the Medical Officer of Health Area, Beruwala, Sri Lanka [15].

\section{Conclusion}

We strongly recommend that interpretation of EBF rates should be done only after careful evaluation of the definitions and survey methods used. As the duration of EBF has been widely discussed over the past few years, a consensus of methods to properly evaluate this indicator should be reached. Programs to strengthen EBF in Sri Lanka remain a priority as the actual rate of EBF to six months in the country is well below the often quoted, but misinterpreted, rate of $75 \%$.

\section{Competing interests}

The authors declare that they have no competing interests.

\section{Authors' contributions}

All authors participated in literature review, conceptualization and drafting of the manuscript and read and approved the final manuscript.

\section{Authors' information}

SBA and TCA are lecturers in Community Medicine in Rajarata University of Sri Lanka with Masters in Community Medicine. Both worked as Community Physicians and have experience in conducting breastfeeding research, promotion and training. SBA is currently a doctoral student and attached to the Post Graduate Institute of Medi- cine, Colombo, Sri Lanka. AdS is a BSc graduate in Biomedical Science and a public health volunteer worker in the field practice area of the Faculty of Medicine and Allied Sciences, Rajarata University of Sri Lanka.

\section{Acknowledgements}

We acknowledge the support given by Prof. D. B. Nugegoda in drafting this manuscript.

\section{References}

I. Department of Census and Statistics: Sri Lanka Demographic and Health Survey 2006/7 Preliminary Report (Draft). Department of Census and Statistics In collaboration with Ministry of Health care and Nutrition ed. Colombo; 2008.

2. International Baby Food Action Network: The state of the world's breastfeeding, Sri Lankan report card. New Delhi, India: IBFAN Asia Pacific; 2007.

3. Labbok M, Krasovec K: Toward consistency in breastfeeding definitions. Stud Fam Plann 1990, $21: 226-230$.

4. Labbok $\mathrm{MH}$, Coffin CJ: A call for consistency in definition of breastfeeding behaviors. Soc Sci Med 1997, 44:1931-1932.

5. World Health Organization: Indicators for Assessing Infant and Young Child Feeding Practices: Part I Definitions Geneva, Switzerland: World Health Organization; 2008.

6. Webb K, Marks GC, Lund-Adams M, Rutishauser IH, Abraham B: Towards a national system for monitoring breastfeeding in Australia: recommendations for population indicators, definitions and next steps. Australian Food and Nutrition Monitoring Unit 2001.

7. Piwoz EG, Creed de Kanashiro H, Lopez de Romana G, Black RE, Brown KH: Potential for misclassification of infants' usual feeding practices using 24-hour dietary assessment methods. J Nutr 1995, I 25:57-65.

8. Zohoori N, Popkin BM, Fernandez ME: Breast-feeding patterns in the Philippines: a prospective analysis. J Biosoc Sci 1993, 25: $127-138$

9. Aarts C, Kylberg E, Hornell A, Hofvander Y, Gebre-Medhin M, Greiner T: How exclusive is exclusive breastfeeding? A comparison of data since birth with current status data. Int J Epidemiol 2000, 29: I04 I-1046.

10. Ssenyonga R, Muwonge R, Nankya I: Towards a better understanding of exclusive breastfeeding in the era of HIVIAIDS: a study of prevalence and factors associated with exclusive breastfeeding from birth, in Rakai, Uganda. J Trop Pediatr 2004, 50:348-353.

II. Engebretsen IM, Wamani H, Karamagi C, Semiyaga N, Tumwine I, Tylleskar T: Low adherence to exclusive breastfeeding in Eastern Uganda: a community-based cross-sectional study comparing dietary recall since birth with 24 -hour recall. $B M C$ Pediatr 2007, 7: 10.

12. Agampodi SB, Fernando S, Dharmaratne SD: Comparison of definitions used for exclusive breast feeding-the Labbock's strict definition and the WHO definition as methods of estimating exclusive breastfeeding rates in Sri Lanka. Ceylon Medical Journal 2009, 54(SuppI I):20.

13. Agampodi SB, Agampodi TC, Piyaseeli UK: Breastfeeding practices in a public health field practice area in Sri Lanka: a survival analysis. Int Breastfeed J 2007, 2:13.

14. Agampodi SB, Fernando S, Dharmaratne SD: Prevalence of undesirable infant feeding practices in a rural community in Matale district- a preliminary analysis. Ceylon Medical Journal 2009, 54(suppl I):57.

15. Agampodi SB, Agampodi TC: Effect of low cost public health staff training on exclusive breastfeeding. Indian J Pediatr 2008, 75:1115-1119. 